\title{
Theory of metastable state relaxation in a gravitational field for non-critical binary systems with non-conserved $0306 / 4$ order parameter
}

\author{
Alexander F Izmailov and Allan S Myerson \\ Department of Chemical Engineering, Polytechnic University, Six Metrotech Center, 333 \\ Jay Street, Brooklyn, New York 11201, USA
}

Received 12 June 1992

\begin{abstract}
A new mathematical ansatz is developed for solution of the time-dependent Ginzburg-Landau nonlinear partial differential equation describing metastable state relaxation in binary (solute + solvent) non-critical solutions with non-conserved scalar order parameter in presence of a gravitational field. It has been demonstrated analytically that in such systems metastability initiates heterogeneous solute redistribution which results in the formation of a non-equilibrium singly-periodic spatial solute structure in the new solute-rich phase. The critical radius of nucleation and the induction time in these systems are gravity-dependent. It has also been proved that metastable state relaxation in vertical columns of supersaturated non-critical binary solutions leads to formation of the solute concentration gradient. Analytical expression for this concentration gradient is found and analysed. $1 t$ is concluded that gravity can initiate phase separation (nucleation or spinodal decomposition).
\end{abstract}

\section{Introduction}

Experimental study of the metastable state relaxation in binary solutions is of great scientific and industrial interest. In these experiments the usual routine is to investigate different stages of the growth process of stable solute nuclei and phenomena associated with this growth [1-3]. Metastable state relaxation is not only the growth of nuclei which is very rapid but also solution ordering. In this relaxation process very little is known about the metastable stage preceding nucleation (MSPN). MSPN manifests itself in solute fluctuations and redistributions which lead to formation of the critical solute nuclei. The MSPN lifetime is known as induction time $t_{\mathrm{c}}$ [1-3]. Recently, experimental studies of MSPN in vertical columns filled with supersaturated solutions have provided interesting data on solute sedimentation [4-8]. In these experiments, initially undersaturated solutions (usually aqueous solutions) have been subjected to a temperature quench process which transfers the system to a metastable state. Having carried out this transfer it is possible to observe a solute sedimentation process which manifests itself in formation of the solute concentration gradients along vertical columns filled with the supersaturated solutions. It has been observed that the solute concentration gradient appears in vertical columns only at the temperatures corresponding to saturated and supersaturated solutions. These experiments dealing with formation of the solute concentration gradient in MSPN are of great interest since they help to provide information about the nature of MSPN by examining solute sedimentation. 
In this introductory section let us describe qualitatively physics of the solute sedimentation phenomenon which takes place in vertical columns filled with supersaturated binary solutions. Metastable state relaxation is characterized by an induction time $t_{\mathrm{c}}$. This time depends on the depth of penetration into metastable region and on the solution purity: in the case of little dust and dirt, induction time can be significantly increased. In this situation it is possible to investigate different kinetic phenomena in the MSPN. The only restriction of such investigations is associated with the characteristic times of the process under consideration. For example, in our case of the solute sedimentation process its characteristic time (sedimentation time) $t_{\mathrm{s}}$ has to be:

(a) less than or equal to the induction time $\left(t_{\mathrm{s}} \leqslant t_{\mathrm{c}}\right)$.

(b) sufficiently long for a noticeable solute concentration gradient to appear.

At present two attempts to explain this phenomenon have been made $[9,10]$. The first one [9] proves that no reasonable value for $t$, can be obtained within the formalism of classical non-equilibrium thermodynamics. It has been concluded in this paper that the gravitational and buoyancy forces cannot alone be responsible for the solute sedimentation process. In paper [10] we have concluded that this process can be understood as the redistribution of the solute subcritical nuclei in the presence of a gravitational field. MSPN is characterized by the situation when new-born solute aggregates (nuclei) are subcritical, i.e. it is energetically favourable for these aggregates to dissolve rather than to grow. In its turn, as it has been demonstrated in [10], the probability for these solute subcritical nuclei to appear depends on the density of their potential energy $h g$ in a gravitational field, where $h$ is the height of the location of the solute aggregate under consideration and $g$ is the free-fall acceleration. Thus, it has been derived in [10] that due to the non-linear character of interaction between a gravitational field and the field variable describing solute concentration, the probability of birth of the subcritical solute nucleus on column bottom is greater than the same probability at the top. The only restriction imposed on the theoretical approach presented in [10] is that the supersaturated solution has a metastable state which is close to the coexistence (binodal) line. In the present paper this restriction will be eliminated.

\section{Statement of the problem}

In this paper we will consider an inhomogeneous irreversible process describing the relaxation from a non-equilibrium metastable state to an equilibrium stable state in the presence of a gravitational field. Equations describing such relaxation processes in time are known in the literature $[11,12]$ as the time-dependent Ginzburg-Landau (TDGL) equations. They are formulated in terms of the local order parameter field $\varphi(r, t)$, where $r$ is its spatial location and $t$ is the current time. In our problem, the role of this order parameter field can be played by the averaged local solute concentration in the new solute-rich phase. At a time just after a temperature quench (transfer into a metastable state) there is yet no new solute-rich phase. However, since the system under consideration is already in a metastable state there is a stochastic birth process of subcritical solute-rich nuclei. These nuclei born due to thermal fluctuations are supposed to dissolve in the course of the relaxation process until the moment when spontaneous nucleation takes place. At that moment the characteristic size of the nucleus (its surface radius, for example) becomes equal to or greater than the critical size $r_{\mathrm{c}}$. The time characterizing this moment is the induction time $t_{\mathrm{c}}$ mentioned 
previously. At that time the probability of finding at least one critical solute-rich nucleus becomes equal to unity. Thus, induction time characterizes the moment when critical and supercritical solute-rich nuclei begin to appear in a solution. These nuclei are supposed to grow in the course of the relaxation since it is energetically more favourable. Thus, the order parameter $\varphi(r, t)$ corresponding to solute concentration in the new solute-rich phase is subjected to small positive changes around zero due to subcritical nuclei until the moment of spontaneous nucleation when it begins to grow rapidly. This means that the order parameter is a non-conserved quantity with respect to metastable state relaxation:

$$
\frac{\mathrm{d}}{\mathrm{d} t} \int_{V} \mathrm{~d}^{3} r \varphi(r, t) \neq 0
$$

where $V$ is the solution volume. In addition we have to note that for the case of the binary solutions order parameter, $\varphi(\boldsymbol{r}, t)$ is a scalar function.

Simple theoretical consideration of the situation described above for the spherically symmetrical case deals with concept of the minimum work $E_{\min }\left(r_{\mathrm{s}}\right)$ needed to form a solute nucleus of radius $r_{s}$ :

$$
E_{\min }\left(r_{\mathrm{s}}\right)=E_{\mathrm{s}} r_{\mathrm{s}}^{2}+E_{h} r_{\mathrm{s}}^{3}
$$

where the first term describes the energy increase associated with the positive energy change due to surface formation and the second term is associated with the change of bulk energy. It is assumed also that there exists the distribution function $W\left(r_{\mathrm{s}}\right)$ of solute-rich nuclei with respect to their size $r_{\mathrm{s}}$. Generally speaking, this function depends not only on the thermodynamic state of a solution but also on the nuclei flux $J$. It is convenient together with distribution function $W\left(r_{\mathrm{s}}\right)$ to introduce the pseudoequilibrium distribution function $W_{\mathrm{eq}}\left(r_{\mathrm{s}}\right)$ for the hypothetical case when there is a detailed thermodynamic equilibrium between nuclei dissolution and growth processes (in this case $J=0)$. There is the following expression for function $W_{\mathrm{cq}}\left(r_{\mathrm{s}}\right)[11,12]$ :

$$
W_{\text {eq }}\left(r_{\mathrm{s}}\right)=\text { const } \cdots \exp \left\{-E_{\min }\left(r_{\mathrm{s}}\right) / k T\right\}
$$

where $T$ is the solution temperature. On the basis of these results obtained within the classical non-equilibrium thermodynamics it has been concluded by Volmer, Weber [13] and Frenkel [14] that the metastable state lifetime $t_{\mathrm{c}}$ is proportional to the following Boltzmann's factor:

$$
t_{\mathrm{c}}=\frac{1}{W\left(r_{\mathrm{c}}\right)} \quad W\left(r_{\mathrm{c}}\right)=\text { const } \cdot \exp \left\{-E_{\min }\left(r_{\mathrm{c}}\right) / k T\right\} .
$$

It is understandable that the above presented discussions are valid only for the homogeneous state. However, taking into account the influence of a gravitational field on the metastable state relaxation requires us to consider a heterogeneous case. This means that all characteristics of the metastable state relaxation process such as induction time $t_{\mathrm{c}}$ and critical radius $r_{\mathrm{c}}$ become dependent on the density of nuclei potential energy $h g$ in a gravitational field.

A metastable state relaxation equation in terms of the order parameter $\varphi(r, t)$ was introduced by Ginzburg, Landau [15] and Cahn, Hillard [16]. This equation, known at present as the TDGL equation, has the following form:

$$
\frac{\partial \varphi(\boldsymbol{r}, t)}{\partial t}=-\Gamma \frac{\delta F_{\varphi}(t)}{\delta \varphi(\boldsymbol{r}, t)}+\gamma(\boldsymbol{r}, t) \quad F_{\varphi}(t)=\frac{1}{V} \int_{V} \mathrm{~d}^{3} r F[\varphi(\boldsymbol{r}, t)]
$$


where $\mathrm{I}>0$ is the so-called Landau-Khalatnikov damping coefficient which sets the time scale of relaxation process [15] and functional $F[\varphi(r, t)]$ is the Gibbs free energy density for the configuration characterized by the order parameter $\varphi(r, t)$. The random function $\gamma(\boldsymbol{r}, t)$ represents the Gaussian thermal noise which satisfies the fluctuationdissipation theorem $[17,18]$ :

$$
\begin{aligned}
& \langle\gamma(\boldsymbol{r}, t)\rangle=0 \\
& \left\langle\gamma\left(\boldsymbol{r}_{1}, t_{1}\right) \gamma\left(\boldsymbol{r}_{2}, t_{2}\right)\right\rangle=\frac{(2 \pi)^{3}}{V_{\mathrm{r}}} \Gamma \delta\left(\boldsymbol{r}_{1}-\boldsymbol{r}_{2}\right) \delta\left(t_{1}-t_{2}\right)
\end{aligned}
$$

where $V_{\mathrm{r}}$ is the volume in the reciprocal space, i.e. in the space of Fourier images. Thus, by relations $(4)$ and $(5 a, 5 b)$ we have determined the Langevin equation. In terms of this equation it is possible to distinguish the slow and fast changing degrees of freedom: the order parameter $\varphi(r, t)$ is the slow-changing hydrodynamic degree of freedom whereas the random function $\gamma(r, t)$ can be treated as the fast-changing degree of freedom which plays the role of a thermal bath. This means that the fast-changing degrees of freedom come to almost immediate equilibrium and are described by the Gibbs distribution for any given configuration of the slow-changing hydrodynamic degree of freedom $\varphi(r, t)$. The latter, according to equation (4), relaxes toward the stable state via the formation of nuclei of the new solute-rich phase.

Since the Gibbs time-dependent free energy functional $F_{\varphi}(t)$ in equation (4) does not depend on relaxation dynamics we adopt, for its density, the standard GinzburgLandau expression [15]:

$$
F[\varphi(r, t)]=\frac{K}{2}[\nabla \varphi(r, t)]^{2}+f[\varphi(x, t)]
$$

where the coefficient $K$ gives the range over which spatial inhomogeneities persist and the functional $f[\varphi(r, t)]$ is the uniform potential density in the field of which the scalar order parameter $\varphi(r, t)$ evolves. Let us consider the case of non-critical solutions, i.e. solutions which do not possess critical points or such solutions where critical points are hardly achievable. Partial thermodynamic equilibrium at metastable states of these solutions can be characterized at any time instant by the time-dependent Gibbs free energy functional $F_{\varphi}(t)$. The simplest form for the potential density $f[\varphi(r, t)]$ of this functional in the case of non-critical solutions in a gravitational field can be presented as follows:

$$
f[\varphi(\boldsymbol{r}, t)]=\frac{\mu}{2} \varphi^{2}(\boldsymbol{r}, t)-\frac{\eta}{3} \varphi^{3}(\boldsymbol{r}, t)-\frac{h g}{\Gamma D} \varphi(\boldsymbol{r}, t)
$$

where $\mu$ and $\eta$ are the positive coefficients characterizing depth of penetration into metastable state, $D=\Gamma K$ is the diffusion coefficient and $h g$ is the density of potential energy in the gravitational field for the given order parameter $\varphi(\boldsymbol{r}, t)$ configuration (positive direction of the column height is from the column top to its bottom). Now one can rewrite equation (4) in the form of the nonlinear inhomogeneous partial differential equation:

$$
\frac{\partial \varphi(\boldsymbol{r}, t)}{\partial t}=D \nabla^{2} \varphi(\boldsymbol{r}, t)-\Gamma \mu \varphi(\boldsymbol{r}, t)+\Gamma \eta \varphi^{2}(\boldsymbol{r}, t)+\gamma(\boldsymbol{r}, t)+\frac{h g}{D} .
$$

This equation can be treated as one of the possible modifications of the general TDGL equation [15]. The simple analysis of metastability indicates that it can be obtained 
only for the order parameter $\varphi(r, t)$ values from the following interval:

$\varphi_{\mathrm{s}}\left[1-(1-\xi(h g))^{1 / 2}\right]<\varphi(r, t)<\varphi_{\mathrm{s}} \quad \varphi_{\mathrm{s}}=\frac{\mu}{2 \eta} \quad \xi(h g)=\frac{4 h g \eta}{\Gamma D \mu^{2}} \leqslant 1$.

It is noteworthy that gravity can induce spinodal decomposition when $h g \simeq \Gamma D \mu^{2} / 4 \eta$. In this case, width of the metastable region is negligibly small and any temperature quench brings the system directly to the region of unstable states which immediately phase separate. For the order parameter $\varphi(r, t)$ values which are greater than $\varphi_{\mathrm{s}}$ we also obtain the solution unstable states. These states cannot exist in nature like the uniform states and phase separate through the spinodal decomposition mechanism. In addition, result (9) gives us the natural restriction imposed on parameters of the TDGL equation describing metastable state relaxation in a gravitational field:

$$
\xi(h g) \leqslant 1 \text {. }
$$

Thus, with the help of equation (8) one can describe metastable state relaxation in a gravitational field for the case of binary non-critical solutions. One of the main goals we are striving for in this paper is to determine how the metastable state lifetime $t_{\mathrm{c}}$ depends on gravity: $t_{\mathrm{c}}=t_{\mathrm{c}}(h g)$ in the particular case when solution supersaturation is kept unchanged up to the moment of nucleation onset. Exactly this situation has been investigated in experimental studies [4-8].

\section{Solution of the metastable state relaxation problem}

In this paper we are developing a new ansatz for solution of nonlinear inhomogeneous partial differential equation (8). For this purpose let us rewrite equation (8) in the equivalent form of the following system of two differential equations:

$$
\begin{aligned}
& \frac{\partial \varphi(\boldsymbol{r}, t)}{\partial t}=2 D\left[\frac{1}{r}-\frac{A(h g)}{r_{\mathrm{c}}(h g)}\right] \frac{\partial \varphi(\boldsymbol{r}, t)}{\partial r}+\frac{h g}{D}+\gamma(\boldsymbol{r}, t) \quad r=|\boldsymbol{r}| \\
& D \nabla^{2} \varphi(\boldsymbol{r}, t)-2 D\left[\frac{1}{r}-\frac{A(h g)}{r_{\mathrm{c}}(h \boldsymbol{g})}\right] \frac{\partial \varphi(\boldsymbol{r}, t)}{\partial r}+\Gamma \mu \varphi(\boldsymbol{r}, t)-\Gamma \eta \varphi^{2}(\boldsymbol{r}, t)=0
\end{aligned}
$$

where $r_{\mathrm{c}}=r_{\mathrm{c}}(h g)$ is the gravity-dependent critical radius of nucleation which is timeindependent in the problem of constant supersaturation. The gravity-dependent auxiliary function $A(h g)$ will be defined later.

The mathematical ansatz for solution of system $(11 a, 11 b)$ of differential equations can be given in the form of the following scheme:

(1) We start solution of equation system $(11 a, 11 b)$ from the solution of equation ( $11 b)$. Let us try to find the spherically symmetrical solution of this equation in the form:

$$
\varphi(r, t)=\varphi(r, t)=\alpha \chi[r(t)] \quad r(t)=\left[r-\frac{1}{\lambda} \frac{r_{\mathrm{s}}^{\lambda}(t)}{r_{\mathrm{c}}^{\lambda-1}}\right]
$$

where $\alpha$ and $\lambda$ are the constants to be determined and $r_{s}(t)$ is the time-dependent radius of the evolving embryo surface. Substitution of this expression for the spherically symmetrical order parameter $\varphi(r, t)$ into equation $(11 b)$ allows one to rewrite it in the form:

$$
\frac{\mathrm{d}^{2} \chi[r(t)]}{\mathrm{d} r^{2}(t)}+2 \frac{A(h g)}{r_{\mathrm{c}}(h g)} \frac{\mathrm{d} \chi[r(t)]}{\mathrm{d} r(t)}+\frac{\Gamma \mu}{D} \chi[r(t)]-\frac{\Gamma \eta \alpha}{D} \chi^{2}[r(t)]=0 .
$$


As it has been shown by Painleve [19] this ordinary nonlinear homogeneous differential equation of the second order can possess solution only under the following conditions:

$$
2 \frac{A(h g)}{r_{\mathrm{c}}(h g)}=5 d \quad \frac{\Gamma \mu}{D}=6 d^{2} \quad \frac{\Gamma \eta \alpha}{D}=6 .
$$

These conditions allow us to express gravity-dependent critical radius of nucleation $r_{c}(h g)$ together with constants $d$ and $\alpha$ through the gravity-dependent function $A(h g)$ and parameters $\mu$ and $\eta$ of the potential energy density $f[\varphi(r, t)]$ :

$$
\begin{aligned}
& r_{\mathrm{c}}(h g)=\frac{2}{5} A(h g) \sqrt{\frac{6 D}{\Gamma \mu}} \quad d=\frac{2 A(h g)}{5 r_{\mathrm{c}}(h g)}=\sqrt{\frac{\Gamma \mu}{6 D}} \\
& \alpha=\frac{6 D}{\Gamma \eta}=\frac{25}{4}\left(\frac{\mu}{\eta}\right)\left[\frac{r_{\mathrm{c}}(h g)}{A(h g)}\right]^{2} .
\end{aligned}
$$

Under conditions (14) equation (13) acquires the following form:

$$
\frac{\mathrm{d}^{2} \chi[r(t)]}{\mathrm{d} r^{2}(t)}+5 \mathrm{~d} \frac{\mathrm{d} \chi[r(t)]}{\mathrm{d} r(t)}+6 \mathrm{~d}^{2} \chi[r(t)]-6 \chi^{2}[r(t)]=0 .
$$

Solution of this equation was given by Painleve [19]:

$$
\chi[r(t)]=\left(\mathrm{d} C_{1}\right)^{2} \mathrm{e}^{-2 \mathrm{~d} r(t)} P\left[C_{1} \mathrm{e}^{-\mathrm{d} r(t)}+C_{2} ; 0,-1\right] .
$$

In this expression $P\left[C_{1} \mathrm{e}^{-\mathrm{d} r(1)}+C_{2} ; 0,-1\right]$ is the doubly-periodic Weierstrassian elliptic function (DPWEF) with invariants $g_{1}=g_{2}=0$ and $g_{3}=-1$ (concerning DPWEF see Appendix and [20]). Arbitrary constants $C_{1}$ and $C_{2}$ of solution (17) have to be determined from the following initial and boundary conditions, respectively:

$$
\begin{aligned}
& \left.\lim _{t \rightarrow 0} X[r(t)]\right|_{r-r_{s}(t)}=0 \\
& \lim _{t \rightarrow 0} \frac{\left.\mathrm{d} X[r(t)]\right|_{r-r_{s}(t)}}{\mathrm{d} r_{\mathrm{s}}(t)}=0 .
\end{aligned}
$$

The meaning of initial condition $(18 a)$ is quite natural: there is no solute-rich phase just after the temperature quench process which brings the system into a metastable state. In its turn, boundary condition (18b) implies that solute concentration changes sharply on the boundary between solute-rich and solute-poor phases. Thus, conditions $(18 a, b)$ help us to conclude that:

$$
C_{1}=z_{0} \exp \left[\mathrm{d} r_{\mathrm{s}}(0)\left[1-\frac{1}{\lambda}\left(\frac{r_{\mathrm{s}}(0)}{r_{\mathrm{c}}(h g)}\right)^{\wedge}\right]\right] \quad C_{2}=0
$$

where $r_{s}(0)$ is the characteristic size of solute unit (molecule or atom) and $z_{0} \approx$ 0.7616479998 is the DPWEF $P[z ; 0,-1]$ zero (for details see in Appendix (A.16, A.17)) :

$$
P\left[z_{0} ; 0,-1\right]=0 .
$$

Now solution (17) can be rewritten as follows:

$$
\chi[r(t)]=\left(\mathrm{d} C_{1}\right)^{2} \mathrm{e}^{2 \mathrm{~d} r(t)} P\left[C_{1} \mathrm{e}^{-\mathrm{d} r(t)} ; 0,-1\right],
$$

where constant $C_{1}$ is given by expression (19). Expression (20) for solution of equation $(11 b)$ is our final result of the first step in our ansatz for the solution to equation (8). 
The most interesting conclusion concerning this solution is that it describes appearance of the gravity-dependent damping spatial periodic structure in the new solute-rich phase during the process of metastable state relaxation. It means that this relaxation occurs in such a way that solute is redistributed periodically from the very beginning of this relaxation process. The time- and gravity-dependent period $\Delta r(t)$ of such spatial heterogeneous structure for solute density can be found from the relation:

$$
C_{1} \exp \left(-\mathrm{d}\left[\Delta r(t)-\frac{1}{\lambda} \frac{r_{s}^{\lambda}(t)}{r_{\mathrm{s}}^{\lambda-1}(h g)}\right]\right)=2 \omega_{2}
$$

where $\omega_{2} \approx 0.9521849997$ is the only real half-period of the DPWEF with invariants $g_{1}=g_{2}=0$ and $g_{3}=-1$ (for details see Appendix (A.1-A.9)). From this relation it is straightforward to obtain that:

$$
\Delta r(t)=r_{\mathrm{s}}(0)+\frac{1}{d} \ln \left(\frac{z_{0}}{2 \omega_{2}}\right)+\frac{1}{\lambda} \frac{r_{\mathrm{s}}^{\lambda}(t)-r_{\mathrm{s}}^{\lambda}(0)}{r_{\mathrm{c}}^{\lambda-1}(h g)} .
$$

In order to analyse the consequences which follow from this expression for the timeand gravity-dependent period of the solute spatial distribution in the new solute rich phase it is natural to assume that initially, at the time instant $t=0$, solute is in the form of elementary solute units with characteristic size $r_{s}(0)$. Taking into account this assumption together with equation (21) one can conclude that the spatial period of the initial solute density distribution in metastable state is gravity-independent:

$$
\Delta r(0)=r_{\mathrm{s}}(0)+\frac{1}{d} \ln \left(\frac{2 \omega_{2}}{z_{0}}\right) \simeq \sum_{2} \ln \left(\frac{5}{2}\right) r_{\mathrm{c}}(0) \approx 2.29072683 r_{\mathrm{c}}(0) .
$$

In this expression we have assumed that $r_{s}(0) \ll r_{\mathrm{c}}(0)$ and for values of $\omega_{2}$ and $z_{0}$ see in Appendix relations (A.9) and (A.17), respectively. This means that initially, just after a quench of a solution into a metastable state, all solute elementary units (for example, molecules or atoms) are already redistributed in such a way that their spatial density is gravity-independent and changes periodically over the entire solution volume. This periodic structure of solute distribution is inherent to metastable states of noncritical solutions: it is initiated by transition into metastable state and is preserved during the following metastable state relaxation process. For the stationary case corresponding to formation of the critical solute nuclei the critical period $\Delta r\left(t_{\mathrm{c}}\right)$ of their spatial distribution becomes gravity-dependent:

$$
\Delta r\left(t_{\mathrm{c}}\right)=r_{\mathrm{s}}(0)+\frac{\varsigma}{2} \ln \left(\frac{5}{2}\right) r_{\mathrm{c}}(0)+\frac{1}{\lambda} \frac{r_{\mathrm{c}}^{\lambda}(h g)-r_{\mathrm{s}}^{\lambda}(0)}{r_{\mathrm{c}}^{\lambda-1}(h g)}
$$

Analysis of this expression will be given later on.

(2) Now let us take into account agreement between two differential equations (11a) and $(11 b)$. Thus, substituting spherically symmetrical expression for the order parameter $\varphi(r, t)$ found from equation $(11 b)$ into equation (11a) one can obtain the following evolution equation for the nucleus surface radius $r_{s}(t)$ :

$$
\frac{d r_{\mathrm{s}}(t)}{\mathrm{d} t}=\left(2 D\left[\frac{A(h g)}{r_{\mathrm{c}}(h g)}-\frac{1}{r}\right]-\frac{h g}{\alpha D} K^{-1}[r(t)]\right)\left[\frac{r_{\mathrm{c}}(h g)}{r_{\mathrm{s}}(t)}\right]^{\lambda-1}+\rho(\boldsymbol{r}, t)
$$

where

$$
\left.\rho(r, t)=-\left[\frac{r_{c}(h g)}{r_{s}(t)}\right]^{\lambda-1}(\alpha K[r(t)]){ }^{1} \gamma(r, t) \quad K[r(t)]=\frac{\mathrm{d} \chi[r(t)]}{\mathrm{d} r(t)}\right) .
$$


In order to investigate evolution of the spherically symmetrical boundary of solute nucleus it is necessary to rewrite equation (24) for $r=r_{s}(t)$ :

$\frac{\mathrm{d} r_{\mathrm{s}}(t)}{\mathrm{d} t}=\left(2 D\left[\frac{A(h g)}{r_{\mathrm{c}}(h g)}-\frac{1}{r_{\mathrm{s}}(t)}\right]-\frac{h g}{\alpha D} K^{\prime 1}\left[r_{\mathrm{s}}(t)\right]\right)\left[\frac{r_{\mathrm{c}}(h g)}{r_{\mathrm{s}}(t)}\right]^{\lambda-1}+\rho\left[r_{\mathrm{s}}(t)\right]$

where

$$
\begin{aligned}
& \rho\left[r_{\mathrm{s}}(t)\right]=-\left[\frac{r_{\mathrm{c}}(h g)}{r_{\mathrm{s}}(t)}\right]^{\lambda-1}\left(\alpha K\left[r_{\mathrm{s}}(t)\right]\right){ }^{\prime} \gamma\left[r_{\mathrm{s}}(t)\right] \\
& K\left[r_{\mathrm{s}}(t)\right]=\left.K[r(t)]\right|_{r=r_{\mathrm{s}}(t) .}
\end{aligned}
$$

Thus, we have obtained the Langevin equation for evolution of the nucleus surface radius $r_{s}(t)$. Random force $\rho\left[r_{\mathrm{s}}(t)\right]$ of this evolution process has the following correlators:

$$
\begin{aligned}
& \left\langle\rho\left[r_{s}(t)\right]\right\rangle=0 \\
& \left\langle\rho\left[r_{s}\left(t_{1}\right)\right] \rho\left[r_{s}\left(t_{2}\right)\right]\right\rangle=D\left[r_{s}\left(t_{1}\right)\right] \delta\left(t_{1}-t_{2}\right)
\end{aligned}
$$

where

$$
D\left[r_{\mathrm{s}}(t)\right]=\Gamma\left[\frac{r_{\mathrm{c}}(h g)}{r_{\mathrm{s}}(t)}\right]^{2(\lambda-1)}\left(\alpha K\left[r_{\mathrm{s}}(t)\right]\right)^{-2}
$$

and averaging is over all possible realizations of the random force $\rho\left[r_{s}(t)\right]$.

Let us now define auxiliary function $A(h g)$ from the condition that at stationary state there exists the following equality:

$$
\lim _{t \rightarrow t_{c}}\left\langle\frac{\mathrm{d} r_{\mathrm{s}}(t)}{\mathrm{d} t}\right\rangle=0
$$

From this condition one can obtain that:

$$
A(h g)=1+\frac{h g r_{\mathrm{c}}(h g)}{2 \alpha D^{2}} K^{-1}\left[r_{\mathrm{c}}(h g)\right] \quad A(0)=1
$$

where

$$
\begin{aligned}
K\left[r_{\mathrm{v}}(h g)\right]= & \lim _{t \rightarrow t_{1}} K\left[r_{\mathrm{s}}(t)\right]=-\mathrm{d}\left(\mathrm{d} C_{1}\right)^{2}\left(2 \boldsymbol{P}\left[C_{1} ; 0,-1\right]+C_{1} \boldsymbol{P}^{(1)}\left[C_{1} ; 0,-1\right]\right)+O(\varepsilon) \\
& \boldsymbol{P}^{\left({ }^{\prime \prime}\right.}\left[C_{1} ; 0,-1\right]=\left.\frac{\mathrm{d} \boldsymbol{P}[z ; 0,-1]}{\mathrm{d} z}\right|_{z=C_{1}} .
\end{aligned}
$$

Now taking into account that function $K\left[r_{\mathrm{s}}(t)\right]$ is the decreasing one with respect to $r_{\mathrm{s}}(t)$ it is possible to conclude that:

(a) when $r_{\mathrm{s}}(t)<r_{\mathrm{c}}(h g)$, then the averaged nucleus surface radius $\left\langle r_{\mathrm{s}}(t)\right\rangle$ is decreasing in the course of the metastable state relaxation $\mathrm{d}\left\langle r_{\mathrm{s}}(t)\right\rangle / \mathrm{d} t<0$;

(b) when $r_{\mathrm{s}}(t)=r_{\mathrm{c}}(h g)$, then the averaged nucleus surface radius $\left\langle r_{\mathrm{s}}(t)\right\rangle$ is kept unchanged $\mathrm{d}\left\langle r_{\mathrm{s}}(t)\right\rangle / \mathrm{d} t=0$ until eventually spontaneous nucleation takes place due to appropriate random thermal fluctuation;

(c) when $r_{\mathrm{s}}(t)>r_{\mathrm{c}}(h g)$, then the averaged nucleus surface radius $\left\langle r_{\mathrm{s}}(t)\right\rangle$ is increasing in the course of the metastable state relaxation $\mathrm{d}\left\langle r_{\mathrm{s}}(t)\right\rangle / \mathrm{d} t>0$.

The last conclusion concerning growth of the averaged nucleus surface radius $r_{\mathrm{s}}(t)$ is due to the nucleation process only and has nothing in common with the coalescence 
process which we do not consider in this paper. Thus, the first term on the right of equation (25) determines the systematic force driving the growth (decay) of solute nucleus of the new solute-rich phase. This force tends to conserve the system uniformity and by itself cannot induce transition into a new phase. Such a transition can be induced only by the second term on the right of equation (25), i.e. by the random force $\rho\left[r_{s}(t)\right]$ which describes thermal fluctuations.

\section{Fokker-Planck formalism and gravity-dependent induction time}

Let us now introduce a statistical description of the system which is governed by equations (25)-(27). A metastable state produced when the equilibrium line of the first-order phase transition is crossed with finite velocity is the state of non-complete equilibrium. In such a state, distribution of the small-scale degrees of freedom ( $r \ll$ $\left.r_{\mathrm{c}}(h g)\right)$, such as thermal fluctuations, corresponds to the local equilibrium. The largescale degrees of freedom $\left(r \geqslant r_{c}(h g)\right)$, such as the scalar order parameter field $\varphi(r, t)$, vary in the course of time in correspondence with equation (11a). The critical nucleus radius given by expressions (15) and (29) depends on the depth of penetration into the region of metastability of the initial phase and on gravity. It has been demonstrated in expression (29) that the last dependence is highly nonlinear.

Metastable state relaxation will be described in this section as relaxation of the size-distribution function $W\left[r_{s}(t)\right]$ for solute nuclei of the new solute-rich phase:

$$
W\left[r_{\mathrm{s}}(t)\right]=\int_{r_{\mathrm{s}}(0)}^{\infty} \mathrm{d} r_{\mathrm{s}}\left(t^{\prime}\right) G\left[r_{\mathrm{s}}(t), r_{\mathrm{s}}\left(t^{\prime}\right)\right] W\left[r_{\mathrm{s}}\left(t^{\prime}\right)\right]
$$

where $G\left[r_{\mathrm{s}}(t), r_{\mathrm{s}}\left(t^{\prime}\right)\right]$ is the transition probability. Following the theory of homogeneous random processes [21] we introduce this probability in the form:

$$
G\left[r_{\mathrm{s}}\left(t_{1}\right), r_{\mathrm{s}}\left(t_{2}\right)\right]=\left\langle\delta\left[r_{\mathrm{s}}\left(t_{1}\right)-r_{\mathrm{s}}\left(t_{2}\right)\right]\right\rangle .
$$

It is well-known $[21,22]$ that when random force in Langevin equation is Gaussian distributed it is possible to construct the stochastically equivalent Fokker-Planck formalism. Within this formalism one is supposed to deal with the linear partial differential equation with respect to the size-distribution function $W\left[r_{\mathrm{s}}(t)\right]$ :

$$
\frac{\partial W\left[r_{\mathrm{s}}(t)\right]}{\delta t}=-\frac{\partial J\left[r_{\mathrm{s}}(t)\right]}{\partial r_{\mathrm{s}}(t)}
$$

where $J\left[r_{\mathrm{s}}(t)\right]$ is the density of flux in the size-space. For this density there is the following expression in terms of Langevin equation (25) characteristics:

$$
J\left[r_{\mathrm{s}}(t)\right]=-B\left[r_{\mathrm{s}}(t)\right] W\left[r_{\mathrm{s}}(t)\right]-D\left[r_{\mathrm{s}}(t)\right] \frac{\partial W\left[r_{\mathrm{s}}(t)\right]}{\partial r_{\mathrm{s}}(t)}
$$

where

$$
B\left[r_{\mathrm{s}}(t)\right]=\left(2 D\left[\frac{1}{r_{\mathrm{s}}(t)}-\frac{A(h g)}{r_{\mathrm{c}}(h g)}\right]+\frac{h g}{\alpha D} K^{\prime}\left[r_{\mathrm{s}}(t)\right]\right)\left[\frac{r_{\mathrm{c}}(h g)}{r_{\mathrm{s}}(t)}\right]^{\lambda-1}
$$

Functions $B\left[r_{\mathrm{s}}(t)\right]$ and $D\left[r_{\mathrm{s}}(t)\right]$ in expression (33) have to be differentiable real functions with the only restriction that $D\left[r_{\mathrm{s}}(t)\right]>0$. The first term on the right of equation (33) describes systematic growth of solute nuclei of the new solute-rich phase

$0378^{\prime \prime} 03119$ 
and is usually called the 'transport term' whereas the second one is responsible for diffusive growth of these nuclei and is called the 'diffusion term'. From the point of view of the Fokker-Planck formalism function $D\left[r_{\mathrm{s}}(t)\right]$ plays the role of the generalized time-dependent diffusion coefficient. It is important to note that this coefficient goes to zero like $r_{s}^{2(1-\lambda)}(t)(\lambda>1)$ in the case when the surface radius $r_{s}(t)$ of solute nucleus tends to infinity. It can be also noted that for the particular case described here diffusion coefficient is related to the probability that a solute elementary unit (molecule or atom) joins the solute-rich nucleus of size $r_{\mathrm{s}}(t)$ per unit time.

The first step in analysis of equation (33) is to find equilibrium distribution function $W_{\mathrm{eq}}\left[r_{\mathrm{s}}(t)\right]$ corresponding to zero flux density $J\left[r_{\mathrm{s}}(t)\right]=0$. This distribution describes the partial thermodynamic equilibrium when nucleus growth and decay processes compensate each other. Thus, it is straightforward to find the following expression for the equilibrium distribution function $W_{\mathrm{eq}}\left[r_{\mathrm{s}}(t)\right]$ :

$$
W_{\mathrm{eq}}\left[r_{\mathrm{s}}(t)\right]=W_{\mathrm{eq}}\left[r_{\mathrm{s}}(0)\right] \exp \left[-\int_{r_{,}(0)}^{r_{(}(t)} \mathrm{d} r \frac{B(r)}{D(r)}\right] .
$$

This function gives the equilibrium distribution of solute-rich nuclei with respect to their size.

In addition to equilibrium solution $W_{\text {eq }}\left[r_{\mathrm{s}}(t)\right]$ one can find the steady state solution $W_{s t}\left[r_{s}(t)\right]$ of equation (33) which corresponds to the constant stationary flux density $J\left[r_{s}(t)\right]=$ constant:

$$
W_{\mathrm{st}}\left[r_{\mathrm{s}}(t)\right]=W_{\mathrm{eq}}\left[r_{\mathrm{s}}(t)\right]\left[1-J_{\mathrm{st}} \int_{r_{\mathrm{s}}(0)}^{r_{(}(t)} \mathrm{d} r \frac{1}{D(r) W_{\mathrm{eq}}(r)}\right]
$$

where

$$
J_{\mathrm{st}}^{\prime}=\int_{r,(0)}^{\infty} \mathrm{d} r \frac{1}{D(r) W_{\mathrm{eq}}(r)}=\frac{1}{W_{\mathrm{eq}}\left[r_{\mathrm{s}}(0)\right]} \int_{r_{\mathrm{s}}(0)}^{\infty} \mathrm{d} r \frac{1}{D(r)} \exp \left[\int_{r_{\mathrm{s}}(0)}^{r} \mathrm{~d} r^{\prime} \frac{B\left(r^{\prime}\right)}{D\left(r^{\prime}\right)}\right] .
$$

The size-independent flux density $J_{\mathrm{st}}$ corresponds to the constant flux density in the stationary nucleation regime. It means that magnitude of this density is the nucleation velocity, i.e. the average change of the nucleus characteristic size per second. Thus, the reverse quantity $J_{\mathrm{st}}^{-1}$ can be associated with the metastable state lifetime $t_{\mathrm{c}}$ :

$$
t_{\mathrm{c}}=J_{\mathrm{st}} \cdot(1 \mathrm{~m})^{-1}
$$

where $1 m$ is the unit length.

The second step in analysis of equation (33) is to find analytical expression for function $W_{\mathrm{eq}}\left[r_{\mathrm{s}}(t)\right]$. In order to carry out this step let us find the interval $\left[K_{0}, K_{\mathrm{c}}\right]$ within which derivative $K\left[r_{\mathrm{s}}(t)\right]$ (for definition see expression (25)) varies when its variable $r_{s}(t)$ changes in the interval $\left[r_{s}(0), r_{\mathrm{c}}(h g)\right]$ (for details concerning derivative of the DPWEF see in appendix expressions (A.12 and A.14)):

$$
\begin{aligned}
& K_{0}=\lim _{r_{(}(t) \rightarrow r_{s}(0)} K\left[r_{\mathrm{s}}(t)\right]=-\left(\mathrm{d} z_{0}\right)^{3} \\
& K_{\mathrm{c}}=\lim _{r_{\mathrm{s}}(t) \rightarrow r_{\mathrm{s}}(h)} K\left[r_{\mathrm{s}}(t)\right]=-\left(\mathrm{d} z_{0}\right)^{3}+O\left[\frac{r_{\mathrm{s}}(0)}{r_{\mathrm{s}}(h g)}\right] .
\end{aligned}
$$

The last equality for $K_{\mathrm{c}}$ is valid only until $r_{\mathrm{c}}(h g) \gg r_{s}(0)$ which is usually the case in nucleation problems. From these expressions for $K_{0}$ and $K_{\mathrm{c}}$ one can conclude that derivative $K\left[r_{\mathrm{s}}(t)\right]$ is almost everywhere constant $\left(K\left[r_{\mathrm{s}}(t)\right] \approx-\left(\mathrm{d} z_{0}\right)^{3}\right)$ when its variable 
$r_{\mathrm{s}}(t)$ changes from $r_{\mathrm{s}}(0)$ to $r_{\mathrm{c}}$. Taking into account this result it is possible to obtain from relations (15) and (29) the following expression for the gravity-dependent critical radius of nucleation $r_{c}(h g)$ :

$$
r_{\mathrm{c}}(h g)=r_{\mathrm{c}}(0)[1+f(h g)]^{-1}
$$

where

$$
f(h g)=\frac{3}{10 z_{0}^{3}} \xi(h g)=0.678714749 \cdot \xi(h g)<1 .
$$

It is obvious from this result that the critical radius of nucleation slightly diminishes in the direction from the column top to its bottom. This decrease becomes more considerable for the metastable states close to spinodal line when $\xi(h g)$ tends to unity (see expression (9)).

Now let us return to the equilibrium size-dependent function $W_{\text {eq }}\left[r_{\mathrm{s}}(t)\right]$. Taking into account results $(38 a, 38 b)$ it is possible to obtain that there is the following expression for the equilibrium distribution of the subcritical $\left(r_{\mathrm{s}}(t) \leqslant r_{\mathrm{c}}(h g)\right)$ solute-rich nuclei with respect to their size:

$$
\begin{aligned}
W_{\mathrm{eq}}\left[r_{\mathrm{s}}(t)\right]= & W_{\mathrm{eq}}\left[r_{\mathrm{s}}(0)\right] \exp \left[-\frac{\alpha\left(\mathrm{d} z_{0}\right)^{3}}{\Gamma r_{\mathrm{c}}^{\lambda-1}(h g)}\right. \\
& \times \int_{r_{s}(0)}^{r_{(}(1)} \mathrm{d} r\left[2 D \alpha\left(\mathrm{d} z_{0}\right)^{3}\left[r^{\lambda-2}-\frac{A(h g)}{r_{\mathrm{c}}(h g)} r^{\lambda-1}\right]-\frac{h g}{D} r^{\lambda-1}\right] \\
= & \exp \left[-\frac{\alpha\left(\mathrm{d} z_{0}\right)^{3}}{\lambda \Gamma}\left(2 D \alpha\left(\mathrm{d} z_{0}\right)^{3}\left[\frac{\lambda}{\lambda-1} R^{\lambda-1}(t, h g)-A(h g) R^{\lambda}(t, h g)\right]\right.\right. \\
& \left.\left.+\frac{h g r_{\mathrm{c}}(h g)}{D} R^{\lambda}(t, h g)\right)\right]
\end{aligned}
$$

where $R(t, h g)=r_{\mathrm{s}}(t) / r_{\mathrm{c}}(h g)$ and function $A(h g)$ is given by expression (15). From this expression it follows that for the equilibrium state under conditions $r_{s}(0) \ll 5 r_{c}(0) / 2$, $r_{s}(t) \leqslant r_{c}(h g)$ and $\lambda=3$, it is possible to restore phenomenological expression (1) for the minimum work $E_{\min }\left(r_{\mathrm{s}}\right)$ needed to form subcritical or critical solute nucleus of the new solute-rich phase with characteristic size $r_{\mathrm{s}}$ :

$$
E_{\min }\left(r_{\mathrm{s}}\right)=E_{\mathrm{s}} r_{\mathrm{s}}^{2}+E_{b} r_{\mathrm{s}}^{3}
$$

where

$$
\begin{aligned}
& E_{\mathrm{s}}=E_{\mathrm{s}}(h g)=\frac{D \alpha^{2}\left(\mathrm{~d} z_{0}\right)^{6}}{\Gamma r_{\mathrm{c}}^{2}(h g)} \\
& E_{b}=E_{b}(h g)=-\frac{2 D \alpha^{2}\left(\mathrm{~d} z_{0}\right)^{6}}{3 \Gamma r_{\mathrm{c}}^{3}(h g)}-\frac{h g \alpha\left(\mathrm{d} z_{0}\right)^{3}}{3 \Gamma D r_{\mathrm{c}}^{2}(h g)} .
\end{aligned}
$$

It is noteworthy that within the developed above theoretical approach we have obtained the correct sign for coefficient $E_{b}$ without special assumptions. Usually the first term of expression (38) is associated with the surface energy: $E_{\mathrm{s}}=4 \pi \sigma$, where $\sigma$ is the surface tension coefficient. Thus, within our approach one can express this coefficient through the TDGL model parameters:

$$
k T \sigma=k T \sigma(h g)=\frac{3 D \alpha^{2}\left(\mathrm{~d} z_{0}\right)^{6}}{4 \pi \lambda \Gamma^{2} r_{c}^{2}(h g)}=\frac{75}{576 \pi \lambda} z_{0}^{6} \frac{\Gamma \mu^{4}}{D \eta^{2}}[1+f(h g)]^{2} .
$$


What concerns height $h$ dependence of the surface tension coefficient $\sigma(h g)$, it can be concluded, that for the fixed gravity acceleration $g$ it grows in the direction from the column top to bottom.

Analysis of result (40) gives that the function $W_{\mathrm{eq}}^{-1}\left[r_{\mathrm{s}}(t)\right]$ has a sharp maximum at $r_{s}(t)=r_{c}(h g)$ which reflects the existence of a nucleation barrier. The expression for this maximum has the form:

$$
W_{\mathrm{eq}}^{1}\left[r_{\mathrm{c}}(h g)\right]=\mathrm{e}^{L_{\lambda}(h g)}
$$

where

$$
\begin{aligned}
L_{\lambda}(h g) & =\frac{2 D \alpha^{2}\left(\mathrm{~d} z_{0}\right)^{6}}{\lambda \Gamma}\left[\frac{\lambda}{\lambda-1}-A(h g)\right]-\frac{h g \alpha\left(\mathrm{d} z_{0}\right)^{3} r_{\mathrm{c}}(h g)}{\lambda \Gamma D} \\
& =\frac{2 D \alpha^{2}\left(\mathrm{~d} z_{0}\right)^{6}}{\lambda \Gamma(\lambda-1)}=\frac{\mu^{3} z_{0}^{6}}{3 \lambda(\lambda-1) \eta^{2}} \sim \frac{1}{r_{\mathrm{c}}(0)} .
\end{aligned}
$$

In order to obtain this result we have used expressions (29) for $A(h g)$ and (38b) for $K\left[r_{\mathrm{c}}(h g)\right]$. It is important to note that function $L_{\lambda}(h g)=L_{\lambda}$ does not depend on gravity.

The third step in analysis of equation (33) is to arrange adequate estimations for the induction time $t_{\mathrm{c}}$. In order to carry out these estimations let us calculate the integral in expression (36):

$$
J_{\mathrm{st}}^{-1}=\frac{1}{W_{\mathrm{eq}}\left[r_{\mathrm{s}}(0)\right]} \int_{r,(0)}^{\infty} \mathrm{d} r \frac{1}{D(r)} \mathrm{e}^{\varepsilon A(r)}
$$

where

$$
\begin{aligned}
& A(r)=\frac{\alpha^{3}}{r_{\mathrm{c}}^{\lambda-1}} \int_{r,(0)}^{r} \mathrm{~d} r^{\prime}\left[\left(\frac{1}{r^{\prime}}-\frac{A(h g)}{r_{\mathrm{c}}(h g)}\right) K\left(r^{\prime}\right)+\frac{h g}{2 D^{2} \alpha}\right]\left(r^{\prime}\right)^{\lambda-1} K\left(r^{\prime}\right) \\
& \varepsilon=\frac{2 D}{\alpha \Gamma}=\frac{\eta}{3} .
\end{aligned}
$$

It can be done with the help of the steepest-descent method [23] which can give correct asymptotic for integral (44) only in the following case: (a) $\varepsilon=\eta / 3 \gg 1$; (b) functions $D^{-1}(r)$ together with $A(r)$ are the real and sufficiently smooth functions of $r$. The classical result for such integrals is due to Laplace who has argued that their main contribution comes from the neighbourhood of the global maximum of function $A(r)$. This maximum takes place at $r=r_{\mathrm{c}}(\boldsymbol{h g})$ and assuming now that $A(r)$ is twice differentiable function it is possible to obtain that:

$$
J_{\mathrm{st}}(h g)^{-1} \simeq\left(-\frac{2 \pi}{\varepsilon A^{(2)}\left[r_{\mathrm{c}}(h g)\right]}\right)^{1 / 2} \frac{1}{D\left[r_{\mathrm{c}}(h g)\right] W_{\mathrm{eq}}\left[r_{\mathrm{c}}(h g)\right]}
$$

where

$$
\begin{aligned}
A^{(2)}\left[r_{\mathrm{c}}(h g)\right] & =\left.\frac{\mathrm{d}^{2} A\left[r_{\mathrm{s}}(t)\right]}{\mathrm{d} r_{\mathrm{s}}^{2}(t)}\right|_{r_{\mathrm{s}}(t)=r_{\mathrm{c}}(h g)} \\
& =-\frac{\alpha^{3}}{r_{\mathrm{c}}^{2}(h g)} K^{2}\left[r_{\mathrm{c}}(h g)\right]-\left.\frac{h g \alpha^{2}}{2 D^{2}} \frac{\mathrm{d} K\left[r_{\mathrm{s}}(t)\right]}{\mathrm{d} r_{\mathrm{s}}(t)}\right|_{r_{\mathrm{s}}(r)=r_{\mathrm{s}}(h g)} \\
D\left[r_{\mathrm{c}}(h g)\right] & =\frac{\Gamma}{\alpha^{2}} K^{-2}\left[r_{\mathrm{c}}(h g)\right] .
\end{aligned}
$$


As it has already been demonstrated there is the following approximate equality:

$$
K\left[r_{\mathrm{c}}(h g)\right] \simeq-\left(\mathrm{d} z_{0}\right)^{3} \quad r_{\mathrm{s}}(0) \leqslant r_{\mathrm{s}}(t) \leqslant r_{\mathrm{c}}(h g)
$$

which is correct if, and only if, condition (38b) is satisfied. Thus, substituting expressions (46-48) into equation (45) one can obtain an expression for the gravitydependent induction time $t_{\mathrm{c}}$ in the form:

$$
t_{\mathrm{c}}=t_{\mathrm{c}}(h g)=t_{\mathrm{c}}(0)[1+f(h g)]^{-1}
$$

where

$$
t_{\mathrm{c}}(0)=\frac{2}{5} z_{0}^{3} \frac{\mu}{\eta}\left(\frac{\pi}{D \Gamma}\right)^{1 / 2} \frac{1}{W_{\mathrm{eq}}\left[r_{\mathrm{c}}(0)\right]} \cdot(1 \mathrm{~m})
$$

In order to obtain expression for stationary flux density $J_{\mathrm{st}}$ in form (48) we have used expressions (15) and (39) for parameters $\alpha, \varepsilon$ and function $r_{\mathrm{c}}(h \mathrm{hg})$, respectively. Relation (48) for induction time demonstrates that this time is gravity-dependent. For the case of the fixed gravity acceleration it is straightforward to conclude that induction time diminishes in the direction from the column top to bottom. Taking into account that maximum value of function $f(h g)$ is equal to $3 / 10 z_{0}^{3} \approx 0.678714749 \cdot(\xi(h g)=1)=$ 0.678714749 (see expression (39)) it is possible to conclude from result (48) that the minimum achievable induction time in nucleation experiments in vertical columns is:

$$
t_{\mathrm{c}, \min }=0.5956938191 \cdot t_{\mathrm{c}}(0) \text {. }
$$

The following decrease of induction time due to gravity is impossible since the following increase of column height at fixed gravity acceleration leads to phase separation on the bottom level of vertical column through spinodal decomposition mechanism (see expression (9) for boundaries of the metastability region). Taking into account relation (9) for $\varphi_{\text {, }}$ one can rewrite expression (48) for induction time $t_{c}(0)$ in the form:

$$
t_{\mathrm{c}}(0)=\frac{4}{5} z_{0}^{3} \varphi_{\mathrm{s}}\left(\frac{\pi}{D \Gamma}\right)^{1 / 2} \frac{1}{W_{\mathrm{eq}}\left[r_{\mathrm{c}}(0)\right]} \cdot(1 \mathrm{~m}) .
$$

Now it is apparent that induction time $t_{\mathrm{c}}(h \mathrm{hg})$ depends not only on gravity but on the depth of penetration into metastable region. To explain that one can distinguish two limiting cases:

(a) $\varphi_{\mathrm{s}} \approx 0$. In this case for the fixed value of $h g$ time $t_{\mathrm{c}}(h g) \approx 0$ what corresponds to the infinitely narrow metastable region (binodal line separates stable and unstable states), i.e. in this case temperature quench brings system almost on the boundary with unstable states which immediately phase separate.

(b) $\varphi, \gg 1$. In this case for the fixed value of $h g$ time $t_{\mathrm{c}}(\mathrm{hg})$ may be very large corresponding to the very narrow unstable region, i.e. in this case the temperature quench always brings a system to a metastable state which can be thought to be very close to the binodal line (to the region of stable states).

All these derived conclusions concerning the induction time $t_{\mathrm{c}}(\mathrm{hg})$ are consistent with the known experimental facts [1-9].

By result (41) we have also obtained restrictions under which the well-known assumptions (2-3) are correct. These assumptions state that there is the inverse proportionality between the induction time $t_{\mathrm{c}}(0)$ and the equilibrium size-distribution function $W_{\mathrm{eq}}\left[r_{\mathrm{c}}(0)\right]$ for critical solute nuclei. This conclusion, $t_{\mathrm{c}}(0) \sim W_{\mathrm{eq}}^{-1}\left[r_{\mathrm{c}}(0)\right]$, was first obtained by Zeldovich [24] on the basis of classical non-equilibrium thermodynamics. In addition it has been proved that the equilibrium size-distribution function $W_{\mathrm{eq}}\left[r_{\mathrm{c}}(h g)\right]$ is gravity-independent: $W_{\mathrm{eq}}\left[r_{\mathrm{c}}(h g)\right]=W_{\mathrm{eq}}\left[r_{\mathrm{c}}(0)\right]$. 


\section{Conclusions}

All results obtained in this paper are due to a new mathematical ansatz for solution of nonlinear partial differential equation (8). This equation describes relaxation in a gravitational field of the non-conserved scalar order parameter $\varphi(r, t)$ which is associated in our paper with solute concentration in the new solute-rich phase. The ansatz is constructed in such a way that equation (8) has been split into two interconnected equations $(11 a)$ and $(11 b)$. The doubly-periodic solution of second equation $(11 b)$ is found and analysed. This solution corresponds to the new topology in solute redistribution within the new solute-rich phase. According to our result (20) this redistribution corresponds to appearance of the damping spatial periodic structure for solute density distribution. The developing periodic structure exists during the metastable state relaxation process until coalescence processes begin to play an essential role. The new heterogeneous topological structure of the solute density distribution is inherent only to the solution metastable state: it appears immediately after solution transformation into metastable state. Since only one period of the found DPWEF is real (see in Appendix expression (A.9)) the solute heterogeneous structure described is in reality a singlyperiodic structure. The main characteristic of such a spatial singly-periodic structure is its period. We have found that this period $\Delta r(t)$ is gravity-and time-dependent (for details see expression (23)).

It is obvious from solution (20) that the metastable state relaxation is also heterogeneous with respect to the density of potential energy in the gravitational field. This fact proves the existence of the solute sedimentation process which takes place in the course of the metastable state relaxation process in a gravitational field up to the moment of spontaneous nucleation onset. Solute sedimentation manifests itself in formation of the solute concentration gradient along the column height. This gradient is directed downward and is associated with contribution to solute density due to solute subcritical nuclei. In order to estimate the solute concentration gradient $\Delta_{H} \varphi\left[r_{\mathrm{s}}(t)\right]=\alpha\left\{\left.\chi\left[r_{\mathrm{s}}(t)\right]\right|_{h=H}-\left.\chi\left[r_{\mathrm{s}}(t)\right]\right|_{h=0}\right\}$, in the vertical column of height $H$ let us assume that for any stage of evolution for the surface radius $r_{\mathrm{s}}(t)$ of solute nucleus there exists the following inequality:

$$
\mathrm{d} r_{\mathrm{s}}(t)\left[1-\frac{1}{\lambda}\left(\frac{r_{\mathrm{s}}(t)}{r_{\mathrm{c}}(h g)}\right)^{\lambda-1}\right] \ll 1
$$

where the surface radius $r_{\mathrm{s}}(t)$ changes in the interval $\left[r_{\mathrm{s}}(0), r_{\mathrm{c}}(h g)\right]$. Under this condition it is possible to expand solution (20) into series around $z_{0}$ (see expression (A.18) in Appendix) and to obtain $\Delta_{H} \varphi\left[r_{\mathrm{s}}(t)\right]$ in the form:

$$
\Delta_{H} \varphi\left[r_{\mathrm{s}}(t)\right]=\frac{\alpha\left(\mathrm{d} z_{0}\right)^{3}}{\lambda r_{\mathrm{c}}^{\lambda-1}(0)}\left[r_{\mathrm{s}}^{\lambda}(t)-r_{\mathrm{s}}^{\lambda}(0)\right]\left[(1+f(H g))^{\lambda-1}-1\right] \text {. }
$$

It is apparent from this expression that the solute concentration gradient is an increasing function of time and gravity. Analysis of expression (51) for $t=t_{\mathrm{c}}(\mathrm{Hg})$ gives that the maximum gradient $\Delta_{H} \varphi\left[r_{\mathrm{c}}(\mathrm{Hg})\right]$ of solute concentration $\varphi\left[r_{\mathrm{s}}(t)\right]$ is the non-trivial function of the potential energy density $\mathrm{Hg}$ in a gravitational field. It is easy to demonstrate that for the case when $\lambda=3$ and $r_{\mathrm{s}}(0) / r_{\mathrm{c}}(0) \ll 1$ this maximum achievable gradient of solute concentration has local maximum with respect to $\mathrm{Hg} \approx(\mathrm{Hg})_{\max } \approx$ $5 z_{0}^{3}(\sqrt{3}-1) \Gamma D \mu^{2} / 6 \eta \approx 0.2696459773 \cdot \Gamma D \mu^{2} / \eta$ :

$$
\left.\Delta_{H} \varphi\left[r_{\mathrm{c}}(H g)_{\max }\right)\right] \approx \frac{4 z_{0}^{3}}{45 \sqrt{3}} \frac{\mu}{\eta} \approx 0.0453681232 \cdot \varphi_{\mathrm{s}} .
$$


Further increase of the potential energy density $\mathrm{Hg}$ leads to the decrease of the achievable solute concentration gradient since gravity-dependent critical radius of nucleation $r_{\mathrm{c}}(\mathrm{Hg})$ and induction time $t_{\mathrm{c}}(\mathrm{hg})$ become sufficiently small for the nucleation process to start. Thus, the rapid nucleation prevents formation of the considerable solute concentration gradient along the column height.

It is also important to note that when column height $H$ is sufficiently large:

$$
H=\frac{5 z_{0}^{3} D \Gamma^{*} \mu^{2}}{6 g \eta}\left[\frac{r_{c}(0)}{r_{s}(0)}-1\right]
$$

it is possible to initiate immediate spontaneous nucleation by gravity. It means that gravity-dependent critical radius of nucleation $r_{\mathrm{c}}(\mathrm{Hg})$ corresponding to column bottom $(h=H)$ becomes equal to the initial characteristic size $r_{\mathrm{s}}(0)$ of solute units (molecules or atoms). In this case nucleation on the bottom level of vertical columns starts immediately after solution transfer into a metastable state.

\section{Appendix: Doubly-periodic Weierstrassian elliptic functions}

Doubly-periodic Weierstrassian Elliptic Function (DPWEF) $P\left(z ; g_{2}, g_{3}\right)$ is an even function of order two which is usually defined by the following double series [15]:

$$
\begin{array}{rlrl}
P\left(z ; g_{2}, g_{3}\right) & =P\left(z \mid \omega, \omega^{\prime}\right)=P\left(-z \mid \omega, \omega^{\prime}\right) \\
& =\frac{1}{z^{2}}+\sum_{m} \sum_{n}\left(1-\delta_{m, 0}\right)\left(1-\delta_{n, 0}\right)\left[\frac{1}{\left(z-\Omega_{m n}\right)^{2}}-\frac{1}{\Omega_{m n}^{2}}\right] & \operatorname{Im}\left(\frac{\omega^{\prime}}{\omega}\right)>0
\end{array}
$$

where

$$
\Omega_{m n}=m 2 \omega+n 2 \omega^{\prime} .
$$

There is also the integral formula for the DPWEF $P\left(z ; g_{2}, g_{3}\right)$ :

$$
z=\int_{P\left(z ; g_{2}, g_{3}\right)}^{x} \mathrm{~d} y\left(4 y_{3}-g_{2} y-g_{3}\right)^{-1 / 2} .
$$

In expression (A.1) $\omega$ and $\omega^{\prime}$ are the DPWEF primitive half-periods; $m$ and $n$ are the integers. Summation in expression (A.1) ranges over all integers $m$ and $n$ except $m=0$ and $n=0$ simultaneously. Double periodicity means that there are the following expressions:

$$
\begin{aligned}
& P\left(z+2 \omega \mid \omega, \omega^{\prime}\right)=P\left(z \mid \omega, \omega^{\prime}\right) \\
& P\left(z+2 \omega^{\prime} \mid \omega, \omega^{\prime}\right)=P\left(z \mid \omega, \omega^{\prime}\right) .
\end{aligned}
$$

The function $P^{\prime}\left(z \mid \omega, \omega^{\prime}\right)=\mathrm{d} P\left(z \mid \omega, \omega^{\prime}\right) / \mathrm{d} z$ is the odd elliptic function of order three with half-periods $\omega_{\alpha}(\alpha=1,2,3)$ :

$$
\omega_{1}=\omega \quad \omega_{2}=-\omega-\omega^{\prime} \quad \omega_{3}=\omega^{\prime} .
$$

It can be concluded that $P^{\prime}\left(\omega_{\alpha} \mid \omega, \omega^{\prime}\right)=P\left(-\omega_{\alpha} \mid \omega, \omega^{\prime}\right)=0$ since $P^{\prime}\left(z \mid \omega, \omega^{\prime}\right)$ has halfperiods $\omega_{\alpha}$ and $P^{\prime}\left(-\omega_{\alpha} \mid \omega, \omega^{\prime}\right)=-P^{\prime}\left(\omega_{\alpha} \mid \omega, \omega\right)$. Thus, it follows that $z=\omega_{\alpha}(\alpha=1,2,3)$ is an irreducible set of zeros for $P^{\prime}\left(z \mid \omega, \omega^{\prime}\right)$. It is customary to put that:

$$
e_{\alpha r}=P\left(\omega_{\alpha} \mid \omega, \omega^{\prime}\right) \quad(\alpha=1,2,3)
$$


The DPWEF invariants $g_{1}, g_{2}$ and $g_{3}$ can be defined in terms of constants $e_{\alpha}(\alpha=1,2,3)$ in the following way:

$$
\begin{aligned}
& g_{1}=e_{1}+e_{2}+e_{3}=0 \\
& g_{2}=2\left(e_{1}^{2}+e_{2}^{2}+e_{3}^{2}\right) \\
& g_{3}=4 e_{1} e_{2} e_{3} .
\end{aligned}
$$

Discriminant $\Delta$ of these relations between $e_{\alpha}$ and $g_{\alpha}(\alpha=1,2,3)$ is given in the form:

$$
\Delta=16\left(e_{1}-e_{2}\right)^{2}\left(e_{1}-e_{3}\right)^{2}\left(e_{2}-e_{3}\right)^{2}=g_{2}^{3}-27 g_{3}^{2} .
$$

In our particular case corresponding to $g_{1}=g_{2}=0$ and $g_{3}=-1$ one can obtain that:

$$
e_{1}=4^{-1 / 3} \mathrm{e}^{i \pi / 3} \quad e_{2}=-4^{-1 / 3} \quad e_{3}=4^{-1 / 3} \mathrm{e}^{-i \pi / 3} .
$$

Now taking into account relations (A.2) and (A.6) it is possible to write the following expression for the real half-period $\omega_{2}$ :

$$
\omega_{2}=V P \int_{e_{2}}^{x} \mathrm{~d} y\left(4 y^{2}+1\right)^{-1 / 2}=\frac{5 \pi}{3^{3 / 2} 2^{5 / 3}} \approx 0.9521849997
$$

where $V P$ is the Cauchy principal value. For non-zero invariants $g_{2}$ and $g_{3}$ there exist the other representations:

$g_{2}=60 \sum_{m} \sum_{n}\left(1-\delta_{m, 0}\right)\left(1-\delta_{n, 0}\right) \Omega_{m n}^{-4} \quad g_{3}=140 \sum_{m} \sum_{n}\left(1-\delta_{m, 0}\right)\left(1-\delta_{n, 0}\right) \Omega_{m n}^{-6}$.

From the definitions presented above one can derive the homogeneity relations for arbitrary $s \neq 0$ :

$$
\begin{aligned}
& P\left(s z \mid s \omega, s \omega^{\prime}\right)=s^{-2} P\left(z \mid \omega, \omega^{\prime}\right) \\
& P\left(s z ; s^{-4} g_{2}, s^{-6} g_{3}\right)=s^{-2} P\left(z ; g_{2}, g_{3}\right)
\end{aligned}
$$

and

$$
\begin{aligned}
& P^{\prime}\left(s z \mid s \omega, s \omega^{\prime}\right)=s^{-3} P^{\prime}\left(z \mid \omega, \omega^{\prime}\right) \\
& P^{\prime}\left(s z ; s^{-4} g_{2}, s^{-6} g_{3}\right)=s^{-3} P^{\prime}\left(z ; g_{2}, g_{3}\right) .
\end{aligned}
$$

Taking into account relation (A.11) and putting $s=i$ one can obtain for the case of negative $g_{3}$ the following result:

$$
P\left(z ; g_{2}, g_{3}\right)=-P\left(i z ; g_{2},-g_{3}\right) \text {. }
$$

Thus, it is always possible to convert the case corresponding to negative $g_{3}$ into the case corresponding to positive $g_{3}$.

Zero $z_{0}$ of the DPWEF is defined through the relation:

$$
P\left(z_{0} ; g_{2}, g_{3}\right)=P\left(z_{0} \mid \omega, \omega^{\prime}\right)=0 \text {. }
$$

At this point there also exists the following useful relation:

$$
P^{\prime}\left(z_{0} ; g_{2}, g_{3}\right)=i \sqrt{g_{3}} \text {. }
$$

Now taking into account integral formula (A.2) for the DPWEF $P\left(z ; g_{2}, g_{3}\right)$ we apparently find $z_{0}$ in the form which corresponds to $g_{2}=0$ and $g_{3}=-1$. We obtain that:

$$
\begin{aligned}
& z_{0}=\int_{0}^{x} \mathrm{~d} y\left(4 y^{3}-g_{2} y-g_{3}\right)^{-1 / 2} . \\
& z_{11}=\int_{0}^{\infty} \mathrm{d} y\left(4 y^{3}+1\right)^{-1 / 2}=\frac{2^{1 / 3} \pi}{3^{3 / 2}} \approx 0.7617479998 .
\end{aligned}
$$


Now it is straightforward to obtain the following expansion of the DPWEF $P\left[z ; g_{2}, g_{3}\right]$ around $z_{0}$ :

$P\left[z ; g_{2}, g_{3}\right]=P^{(1)}\left[z_{0} ; g_{2}, g_{3}\right] u\left[1-3 C_{2} u^{4}+\cdots\right]-\left[5 C_{2}+14 C_{3} u^{2}+\cdots\right]$

where $C_{2}=g_{2} / 20=0$ and $C_{3} / 28=-1 / 28$.

\section{References}

[1] Toshev S 1973 Homogeneous Nucleation in Crystal Growth: An Introduction ed P Hartman (Amsterdam: North-Holland)

[2] Zettlemoyer A C (ed) 1977 Nucleation Phenomena (Amsterdam: Elsevier)

[3] Chernov A A 1984 Modern Crystallography III vol 36 (Springer Series in Solid-State Sciences) (Berlin: Springer)

[4] Mullin J W and Leci C L 1969 Phil. Mag. 191075

[5] Chang Y C and Myerson A S 1984 Industrial Crystallization (Amsterdam: Elsevier)

[6] Larson M A and Garside J 1986 Chem. Eng. Sci. 411285

[7] Veverka $V$ et al 1991 AIChE J. 37490

[8] Ginde R M and Myerson A S 1992 J. Cryst. Growth 11641

[9] Izmailov A F and Myerson A S 1992 J. Cryst. Growth 121723

[10] Izmailoy A F and Myerson A S 1992 Physica 183A 549

[1]] Gunton J D and Droz M 1980 Introduction to the Dynamics of Metastable and Unstable States Lecture Notes in Physics No 132 ed L Garrido (Heidelberg: Springer)

[12] Gunton J D, san Miguel M and Sahni P S 1983 Phase Transitions and Critical Phenomena vol VIII ed $C$ Domb and J Lebowitz (New York: Academic)

[13] Volmer M and Weber A 1926 Z. Phys. Chem. 119277

[14] Frenkel J 1955 The Kinetic Theory of Liquids (New York: Dover)

[15] Ginzburg V L and Landau L D; Landau L D and Khalatnikov I M 1965 Collected Papers of L D Landau ed D ter Haar (London: Pergamon)

[16] Cahn J W and Hillard J E 1959 J. Chem. Phys. 31688

[17] Landau L D and Lifshitz E M 1986 Statistical Physics Course of Theoretical Physics vol 5 (London: Pergamon)

[18] Isihara A 1971 Statistical Physics (New York: Academic)

[19] Painleve P 1902 Acta Marh. 2553

[20] Dutta M and Debnath L 1965 Elements of the Theory of Elliptic and Associated Functions (Calcutta: World Press Private)

[21] Stratonovich R L 1963 Topics in the Theory of Random Noise vol 1 (New York: Gordon and Breach)

[22] Van Kampen N G 1987 Stochastic Processes in Physics and Chemistry (Amsterdam: Elsevier)

[23] Bleistein N and Handelsman R A 1986 Asymptotic Expansion of Integrals (New York: Dover)

[24] Zeldovich Ya B 1943 Ac'ta Physiochem. 181 
. 